University of Windsor

Scholarship at UWindsor

2005

\title{
Binding energy of the positronium negative ion: Relativistic and QED energy shifts
}

Gordon W. F. Drake

University of Windsor

M. Grigorescu

Follow this and additional works at: https://scholar.uwindsor.ca/physicspub

Part of the Physics Commons

\section{Recommended Citation}

Drake, Gordon W. F. and Grigorescu, M.. (2005). Binding energy of the positronium negative ion:

Relativistic and QED energy shifts. Journal of Physics B: Atomic, Molecular and Optical Physics, 38 (18), 3377-3393.

https://scholar.uwindsor.ca/physicspub/49

This Article is brought to you for free and open access by the Department of Physics at Scholarship at UWindsor. It has been accepted for inclusion in Physics Publications by an authorized administrator of Scholarship at UWindsor. For more information, please contact scholarship@uwindsor.ca. 
Binding energy of the positronium negative ion: relativistic and QED energy shifts

This article has been downloaded from IOPscience. Please scroll down to see the full text article.

2005 J. Phys. B: At. Mol. Opt. Phys. 383377

(http://iopscience.iop.org/0953-4075/38/18/009)

View the table of contents for this issue, or go to the journal homepage for more

Download details:

IP Address: 137.207.184.30

The article was downloaded on 26/04/2013 at 15:48

Please note that terms and conditions apply. 


\title{
Binding energy of the positronium negative ion: relativistic and QED energy shifts
}

\author{
G W F Drake and M Grigorescu \\ Department of Physics, University of Windsor, Windsor, Ontario N9B 3P4, Canada \\ E-mail: GDrake@uwindsor.ca
}

Received 1 June 2005, in final form 2 August 2005

Published 5 September 2005

Online at stacks.iop.org/JPhysB/38/3377

\begin{abstract}
The leading relativistic and QED corrections to the ground-state energy of the three-body system $\mathrm{e}^{-} \mathrm{e}^{+} \mathrm{e}^{-}$are calculated numerically using a Hylleraas correlated basis set. The accuracy of the nonrelativistic variational ground state is discussed with respect to the convergence of the energy with increasing size of the basis set, and also with respect to the variance of the Hamiltonian. The corrections to this energy include the lowest order Breit interaction, the vacuum polarization potential, one and two photon exchange contributions, the annihilation interaction and spin-spin contact terms. The relativistic effects and the residual interactions considered here decrease the one-electron binding energy from the nonrelativistic value of $0.01200507023298010769(28)$ au to $0.011981051246(2)$ au $(78831530 \pm 5 \mathrm{MHz})$.
\end{abstract}

\section{Introduction}

The positronium negative ion $\left(\mathrm{Ps}^{-}\right)$is the simplest system composed of three equal mass fermions, $\mathrm{e}^{-} \mathrm{e}^{+} \mathrm{e}^{-}$, bound only by electromagnetic interactions. Similar examples of three-body systems, bound by increasingly complex interactions, are provided by three-quark systems such as the proton and the neutron, and three-nucleon systems, such as the ${ }^{3} \mathrm{H},{ }^{3} \mathrm{He}$ nuclei. The existence of a bound ground state in the $\mathrm{e}^{-} \mathrm{e}^{+} \mathrm{e}^{-}$system was predicted by Wheeler [1] and was observed by Mills [2] by passing a positron beam through a thin carbon film in vacuum. The measured $\mathrm{Ps}^{-} \rightarrow(2 \gamma) \mathrm{e}^{-}$decay rate $\lambda_{\left(\mathrm{Ps}^{-}, 2 \gamma\right)}=2.09(9) \mathrm{ns}^{-1}$ [2] corresponds to a Ps ${ }^{-}$lifetime of $0.478 \mathrm{~ns}$, intermediate between that of para (singlet) Ps (0.125 ns) and ortho (triplet) Ps (140 ns) [3].

The main difficulty encountered in a theoretical description of three-body systems is that in general the nonrelativistic problem is not integrable in either classical or quantum mechanics ${ }^{1}$. In a nonrelativistic approach, accurate numerical approximations to the bound eigenstates of

1 A simple classical solution for systems like $\mathrm{e}^{-} \mathrm{e}^{+} \mathrm{e}^{-}$presumes all particles aligned, in uniform rotation around the positive charge, located at the centre of mass. 
three quantum particles interacting by Coulomb forces can be obtained by the Rayleigh-Ritz variational method. A suitable set of coordinates and basis states for the three-body problem was proposed by Hylleraas [4] during the early days of quantum mechanics, and it was used to calculate the ground-state energy of the helium atom. With respect to this set, the matrix elements of various two-body operators can be expressed in analytical form [5], and extensive high-precision calculations become feasible $[6,7]$.

The relativistic quantum many-body problem can be approached either from the field theory, or by using a Schrödinger equation with an 'action at a distance'-type Hamiltonian, defined by quantizing the classical relativistic system [8]. The field theory approach to the bound state problem leads to a relativistically invariant Bethe-Salpeter equation [9]. In the case of two relativistic electrons, approximate Lorentz invariance is established by introducing the Breit interaction, which can be seen as the quantum correspondent to the Darwin term in classical electromagnetism [10].

In the helium atom, the two electrons move in the Coulomb field created by a composite, heavy nucleus, which to a first approximation can be considered as centre of mass (CM). The case of $\mathrm{Ps}^{-}$is different, because all three particles are elementary, have the same mass and can move to the same degree with respect to the CM.

Numerical calculations for the nonrelativistic ground-state properties of $\mathrm{Ps}^{-}$are presented in [11-15]. The autodetaching states have been studied in [16, 17], while several low-lying resonances have been predicted recently [18], by using a combination of the stochastic variational method (SVM) with correlated Gaussians and the complex scaling method. The photodetachment cross sections have been calculated by Igarashi et al [19] using the hyperspherical close-coupling method.

The accuracy of the $\mathrm{Ps}^{-}$ground-state wavefunctions, given by SVM in a Gaussian basis, was studied by comparison with the direct solution of the Schrödinger equation in [14]. It was shown that despite the fact that in SVM the convergence properties of the expectation values for most operators are better, the wavefunction is less accurate.

The purpose of this work is to present detailed calculations of the lowest order relativistic and quantum electrodynamic corrections to the binding energy of $\mathrm{Ps}^{-}$, relative to Ps. A partial calculation of the relativistic corrections has been published previously [20], but their work needs to be extended and updated. In this work, the accuracy of the $\mathrm{Ps}^{-}$nonrelativistic variational ground state is discussed, considering besides the convergence properties of the energy with the basis size, also the variance of the Hamiltonian. It is shown that in agreement with [14], the variance is larger than the accuracy resulting from convergence. Estimates of the leading relativistic and QED corrections are presented in sections 3 and 4. Tables containing the expectation values of some singular operators appearing in the correction terms, such as $p^{4}$ and delta functions, and the $p^{4}$ terms are given in appendix $\mathrm{A}$. The main results and the concluding remarks are summarized in section 4.

\section{The nonrelativistic quantum three-body problem}

In an arbitrary inertial frame, the intrinsic part of the nonrelativistic Hamiltonian for the quantum three-body system $\mathrm{e}^{-} \mathrm{e}^{+} \mathrm{e}^{-}\left(\right.$or $\left.\mathrm{e}^{+} \mathrm{e}^{-} \mathrm{e}^{+}\right)$is

$$
H_{0}=\left(-\frac{1}{2} \nabla_{13}^{2}-\frac{1}{2} \nabla_{23}^{2}-\frac{1}{2} \nabla_{13} \cdot \nabla_{23}-\frac{1}{r_{13}}-\frac{1}{r_{23}}+\frac{1}{r_{12}}\right) f \text { au, }
$$

where $f=\mu / m, \mu=m / 2$ is the reduced mass, $\nabla_{i j} \equiv \partial / \partial \vec{r}_{i j}, \vec{r}_{i j} \equiv \vec{R}_{i j} / a_{\mu}, \vec{R}_{i j} \equiv \vec{R}_{i}-\vec{R}_{j}$ while $\vec{R}_{i}$ denote the position vectors of the two electrons $(i=1,2)$, and of the positron $(i=3)$. The variables $r_{i j}=\left|\vec{r}_{i j}\right|$ are the relative distances in units of $a_{\mu}=a_{0} / f$, where 
$a_{0}=\hbar^{2} /\left(m e^{2}\right)=5.29177249(24) \mathrm{nm}$ is the Bohr radius. By this choice, the Hamiltonian is naturally expressed in reduced atomic units of energy $f$ au $(=13.6056981(40) \mathrm{eV}=$ 1 Ry for $f=0.5)$, where 1 au $=e^{2} / a_{0}=\alpha^{2} m c^{2}$ is the atomic unit of energy and $\alpha=e^{2} /(\hbar c)=1 / 137.03599911(46)$ is the fine structure constant.

Approximate eigenfunctions of this Hamiltonian are obtained by using the variational method. The trial function is a finite linear combination

$$
\begin{gathered}
\Psi\left(\vec{r}_{13}, \vec{r}_{23} ; \vec{s}_{1}, \vec{s}_{2}, \vec{s}_{3}\right)=\sum_{a, b, c}^{a+b+c \leqslant \Omega} \sum_{p=1}^{3} \sum_{l_{1}, l_{2}}^{l_{1}+l_{2}=L} q_{a b c, p}^{l_{1} l_{2}} \Phi_{l_{1} l_{2} L M}^{a b c, p}\left(\vec{r}_{13}, \vec{r}_{23}\right) \chi_{S_{12} m_{12} \mu_{3}}\left(\vec{s}_{1}, \vec{s}_{2}, \vec{s}_{3}\right) \\
- \text { exchange }(1 \rightleftharpoons 2)
\end{gathered}
$$

of $N_{\mathrm{b}}$ basis elements $\Phi_{l_{1} l_{2} L M}^{a b c, p} \chi_{S_{12} m_{12} \mu_{3}}$. The spacial component $\Phi_{l_{1} l_{2} L M}^{a b c, p}$ is expressed in terms of the correlated Hylleraas coordinates [4]

$$
\Phi_{l_{1} l_{2} L M}^{a b c, p}\left(\vec{r}_{13}, \vec{r}_{23}\right)=r_{13}^{a} r_{23}^{b} r_{12}^{c} \exp \left(-\alpha_{p} r_{13}-\beta_{p} r_{23}\right) \mathcal{Y}_{L M}^{l_{1} l_{2}}\left(\hat{\boldsymbol{r}}_{13}, \hat{\boldsymbol{r}}_{23}\right)
$$

involving products of integral powers $a, b, c$ of all relative distances, and the vector-coupled eigenstates

$$
\mathcal{Y}_{L M}^{l_{1} l_{2}}\left(\hat{\boldsymbol{r}}_{13}, \hat{\boldsymbol{r}}_{23}\right)=\sum_{m_{1}+m_{2}=M} C_{m_{1} m_{2} M}^{l_{1} l_{2} L} Y_{l_{1} m_{1}}\left(\hat{\boldsymbol{r}}_{13}\right) Y_{l_{2} m_{2}}\left(\hat{\boldsymbol{r}}_{23}\right)
$$

of $\vec{L}^{2}$ and $L_{z}$. Here $\hat{r}_{i j}=\vec{r}_{i j} / r_{i j}$ are unit vectors, while $\vec{L}=-\mathrm{i}\left(\vec{r}_{13} \times \nabla_{13}+\vec{r}_{23} \times \nabla_{23}\right)$ is the operator of the intrinsic orbital angular momentum. For the ground state of $\mathrm{Ps}^{-}$, only the basis elements with $l_{1}=l_{2}=L=0$ contribute. The parameter $p$ labels three distinct values of the nonlinear parameters $\alpha_{p}$ and $\beta_{p}$ with $p=1,2,3$. Since each combination of powers $a, b, c$ is thus included three times with different values of $\alpha_{p}$ and $\beta_{p}$, the result is called a 'triple' basis set in Hylleraas coordinates, as described in [13].

The spin function

$$
\chi_{S_{12} m_{12} \mu_{3}}=\sum_{\mu_{1}+\mu_{2}=m_{12}} C_{\mu_{1} \mu_{2} m_{12}}^{\frac{1}{2} \frac{1}{2} S_{12}}\left|\frac{1}{2} \mu_{1}\right\rangle\left|\frac{1}{2} \mu_{2}\right\rangle\left|\frac{1}{2} \mu_{3}\right\rangle
$$

corresponds to the antisymmetric singlet $\left(S_{12}=0\right)$ or symmetric triplet $\left(S_{12}=1\right)$ configurations of the two electrons. By construction, the orbital part then has the opposite exchange symmetry such that the total wavefunction in equation (2) is antisymmetric with respect to the two electrons.

The linear variational coefficients $q_{a b c, p}^{l_{1} l_{2}}$ are found as usual by matrix diagonalization, and the nonlinear parameters $\alpha_{p}, \beta_{p}(p=1,2,3)$ determined by a separate minimization of the energy over the six-dimensional energy surface, as described previously [13]. The action of the operator $-\nabla_{13}^{2} / 2$ on the Hylleraas basis functions is given by

$$
\begin{aligned}
\nabla_{13}^{2} \Phi=\left[\frac{1}{r_{13}^{2}}\right. & \frac{\partial}{\partial r_{13}} r_{13}^{2} \frac{\partial}{\partial r_{13}}+\frac{1}{r_{12}^{2}} \frac{\partial}{\partial r_{12}} r_{12}^{2} \frac{\partial}{\partial r_{12}}-\frac{\vec{l}_{13}^{2}}{r_{13}^{2}} \\
& \left.+\frac{2\left(r_{13}-r_{23} \hat{\boldsymbol{r}}_{13} \cdot \hat{\boldsymbol{r}}_{23}\right)}{r_{12}} \frac{\partial^{2}}{\partial r_{13} \partial r_{12}}-\frac{2}{r_{13} r_{12}} \vec{r}_{23} \cdot \nabla_{13}^{Y} \frac{\partial}{\partial r_{12}}\right] \Phi,
\end{aligned}
$$

where $\vec{l}_{13}=-\mathrm{i} \vec{r}_{13} \times \nabla_{13}$ and $\nabla_{13}^{Y}=-\mathrm{i} \hat{\boldsymbol{r}}_{13} \times \vec{l}_{13}$. A similar expression, obtained by permuting the indices 1 and 2, yields $\nabla_{23}^{2} \Phi$. A simple formula in Hermitian form for an arbitrary matrix element of the Hamiltonian is given by Drake [7].

The accuracy of the wavefunction depends on the dimension $N_{\mathrm{b}}$ of the basis set, as controlled by $\Omega=a+b+c$. As $N_{\mathrm{b}}$ increases, the expectation value of the Hamiltonian 
Table 1. The ground-state expectation values $E_{\mathrm{g}}=\left\langle H_{0}\right\rangle,\left\langle H_{0}^{2}\right\rangle$ and $\sigma^{2}=\left\langle H_{0}^{2}\right\rangle-E_{\mathrm{g}}^{2}$ as a function of the basis dimension $N_{\mathrm{b}}$.

\begin{tabular}{clll}
\hline$N_{\mathrm{b}}$ & $E_{\mathrm{g}}(\mathrm{Ry})$ & $\left\langle H_{0}^{2}\right\rangle\left(\mathrm{Ry}^{2}\right)$ & $\sigma^{2} \times 10^{16}\left(\mathrm{Ry}^{2}\right)$ \\
\hline 324 & -0.52401014041339900028 & 0.274586632449596 & 5194. \\
411 & -0.52401014045555156688 & 0.274586628565868 & 1266. \\
512 & -0.52401014046413904054 & 0.274586627626769 & 318. \\
630 & -0.52401014046566562187 & 0.274586627375932 & 65.1 \\
764 & -0.52401014046591837512 & 0.274586627323102 & 12.0 \\
918 & -0.52401014046595439113 & 0.274586627313704 & 2.55 \\
1089 & -0.52401014046595903866 & 0.274586627311885 & 0.73 \\
1283 & -0.52401014046596000245 & 0.274586627311421 & 0.266 \\
1495 & -0.52401014046596016085 & 0.274586627311222 & 0.067 \\
1733 & -0.52401014046596020319 & 0.274586627311175 & 0.020 \\
1990 & -0.52401014046596021296 & 0.274586627311165 & 0.0096 \\
2276 & -0.52401014046596021482 & 0.274586627311160 & 0.0047 \\
2528 & -0.52401014046596021525 & 0.274586627311158 & 0.0031 \\
Extrap. & $-0.52401014046596021539(3)$ & $0.274586627311156(4)$ & $0.0023(23)$ \\
\hline
\end{tabular}

Table 2. Comparison of variational results for the ground-state nonrelativistic energy of $\mathrm{Ps}^{-}$.

\begin{tabular}{llll}
\hline Method & Reference & $N_{\mathrm{b}}$ & Energy (Ry) \\
\hline Triple Hylleraas & Drake et al [13] & Extrap. & $-0.52401014046596021539(3)$ \\
Triple Hylleraas & Drake et al [13] & 2528 & -0.52401014046596021525 \\
Stochastic & Frolov [15] & Extrap. & $-0.524010140465956(8)$ \\
Stochastic & Frolov [15] & 1600 & -0.524010140465951 \\
Double Hylleraas & Ho [21] & 744 & -0.5240101404657 \\
Hyperspherical & Krivec et al [14] & 676 & -0.5240101390 \\
\hline
\end{tabular}

$\left\langle H_{0}\right\rangle_{\left(N_{\mathrm{b}}\right)}=\left\langle\Psi\left|H_{0}\right| \Psi\right\rangle$ decreases, and in principle, in the limit $N_{\mathrm{b}} \rightarrow \infty$ the series $\left\langle H_{0}\right\rangle_{\left(N_{\mathrm{b}}\right)}$ approaches the exact ground-state energy. An upper limit on the error is provided by the variance $\sigma=\sqrt{\left\langle H_{0}^{2}\right\rangle_{\left(N_{\mathrm{b}}\right)}-\left\langle H_{0}\right\rangle_{\left(N_{\mathrm{b}}\right)}^{2}}$. The variational ground-state energy $E_{\mathrm{g}}=\left\langle H_{0}\right\rangle_{\left(N_{\mathrm{b}}\right)}$ [13] and the present results obtained for $\sigma^{2}$ are given in table 1. The extrapolated energy is $E_{\mathrm{g}}^{\infty}=-0.52401014046596021539(3)$ Ry. Because of the variational stability of $\left\langle H_{0}\right\rangle_{\left(N_{\mathrm{b}}\right)}$ relative to $\left\langle H_{0}^{2}\right\rangle_{\left(N_{\mathrm{b}}\right)}^{2}$, the convergence of $\left\langle H_{0}\right\rangle_{\left(N_{\mathrm{b}}\right)}$ is much better than the variance would indicate. Previous estimates of $E_{\mathrm{g}}$ in $\mathrm{Ps}^{-}$by the correlation-function hyperspherical-harmonic method [14], the stochastic variational method [15] and an earlier version of the present method using a double basis set in Hylleraas coordinates [21] are compared in table 2. The accuracy and efficiency of the triple basis set is evident.

\section{Relativistic corrections}

The quantum description of a relativistic charged fermion is based on the Dirac equation $\mathrm{i} \hbar \partial_{t}\left|\Psi_{D}\right\rangle=\left(\vec{\alpha} \cdot \vec{p} c+\beta m c^{2}+V(\vec{R})\right)\left|\Psi_{D}\right\rangle, \vec{p}=-\mathrm{i} \hbar \partial_{\vec{R}}$. This equation admits two spin- $\frac{1}{2}$ solutions $\Psi_{+}$and $\Psi_{-}$corresponding to the retarded and advanced waves, respectively. A reduction to Schrödinger dynamics in the nonrelativistic Hilbert space makes sense only to the extent that $\left|\Psi_{D}\right\rangle$ can be expressed as a product $\Psi_{b} \otimes|b\rangle_{\tau}$ between a 'Pauli-spin-orbit' component $\Psi_{b}(\vec{R}, \vec{s})$, and a 'Dirac-spin" component $|b\rangle_{\tau}, \beta|b\rangle_{\tau}=b|b\rangle_{\tau}, b= \pm$. For a free 
particle $(V=0)$ this factorization is obtained after a Foldy-Wouthuysen (FW) transformation [22]

$$
U_{0}\left(\vec{\alpha} \cdot \vec{p} c+\beta m c^{2}\right) U_{0}^{-1}=\beta H
$$

with

$$
U_{0}(\mathbf{p})=\frac{m c^{2}+H+\beta \alpha \cdot \mathbf{p} c}{\sqrt{2 H\left(m c^{2}+H\right)}}
$$

and

$$
H=\sqrt{p^{2} c^{2}+m^{2} c^{4}}, \quad p=|\vec{p}| .
$$

The relativistic corrections for the case of a bound system of three interacting fermions were first discussed by Stone [23], and the various terms calculated in detail by Drake [6] in connection with the isotope shift for helium. An FW transformation of the Hamiltonian

$$
H_{D}=\sum_{i=1}^{3} \vec{\alpha}_{i} \cdot \vec{p}_{i} c+\beta_{i} m_{i} c^{2}+V\left(\vec{R}_{1}, \vec{R}_{2}, \vec{R}_{3}\right)
$$

can be used to identify the equivalent nonrelativistic operators whose expectation values give the lowest order relativistic corrections to the low-lying energy levels. Consider the change of representation provided by the unitary operator

$$
U=\Pi_{i=1}^{3} U_{0}\left(\mathbf{p}_{i}\right) .
$$

An evaluation of $H_{D}^{\prime}=U H_{D} U^{-1}$ as a power series up to the fourth order in $v / c$ yields $H_{D}^{\prime} \approx H_{\alpha}+H_{\beta}+H_{V}$, where

$$
\begin{aligned}
& H_{\alpha}=\sum_{i=1}^{3} \frac{\beta_{i} \alpha_{i}}{2 m_{i} c} \cdot\left(\left[\mathbf{p}_{i}, V\right]+\sum_{j>i} \frac{\beta_{j}}{2 m_{j} c}\left[\alpha_{j} \cdot \mathbf{p}_{j},\left[\mathbf{p}_{i}, V\right]\right]\right), \\
& H_{\beta}=\sum_{i=1}^{3} \beta_{i}\left(m_{i} c^{2}+p_{i}^{2} / 2 m_{i}-p_{i}^{4} / 8 m_{i}^{3} c^{2}\right), \\
& H_{V}=V-\sum_{i=1}^{3} \frac{1}{8 m_{i}^{2} c^{2}}\left\{\left[\mathbf{p}_{i} \cdot,\left[\mathbf{p}_{i}, V\right]\right]-4 \hbar \mathbf{s}_{i} \cdot\left(\frac{\partial V}{\partial \mathbf{R}_{i}} \times \mathbf{p}_{i}\right)\right\} .
\end{aligned}
$$

Moreover, at the $(v / c)^{4}$ level of accuracy the residual term $H_{\alpha}$ of order $(v / c)^{3}$ can be neglected, because the contributions of the part linear in $\alpha_{i}$ from $H_{D}^{\prime}$ to the energy levels of $H_{\beta}+H_{V}$ are second order $\left((v / c)^{6}\right)$, or higher in the perturbation series. With these assumptions, the general solution of the eigenvalue equation

$$
H_{D}^{\prime}\left|\Psi_{D}\right\rangle=E_{D}\left|\Psi_{D}\right\rangle
$$

is

$$
\left|\Psi_{D}\right\rangle=\Psi_{b_{1} b_{2} b_{3}}\left(\vec{R}_{1} \vec{s}_{1}, \vec{R}_{2} \vec{s}_{2}, \vec{R}_{3} \vec{s}_{3}\right)\left|b_{1} b_{2} b_{3}\right\rangle_{\tau}, \quad b_{i}= \pm .
$$

The $\mathrm{e}^{-} \mathrm{e}^{+} \mathrm{e}^{-}$system has a rest mass $\sim 3 m$, so that we should take the physical ground state of the form $\left|\Psi_{D}\right\rangle=\Psi(1,2,3)|+++\rangle_{\tau}$. This corresponds to the energy $E_{D}=3 m c^{2}+E$, where $E$ is the eigenvalue of the equation $H \Psi=E \Psi$ for the Hamiltonian $H=H_{\mathrm{CM}}^{0}+H_{0}+H_{1}+H_{2}$, in which $H_{0}$ is the intrinsic term given by equation (1),

$$
H_{1}=-\frac{1}{8 m^{3} c^{2}}\left(p_{1}^{4}+p_{2}^{4}+p_{3}^{4}\right)
$$


takes into account the variation in the mass of the constituents with the intrinsic velocity $(v \sim \alpha c)$ and

$$
H_{2}=-\frac{1}{8 m^{2} c^{2}} \sum_{i=1}^{3}\left[\vec{p}_{i} \cdot\left[\vec{p}_{i}, V\right]\right] .
$$

The term $H_{\mathrm{CM}}^{0}=P_{0}^{2} / 6 m, \vec{P}_{0}=-\mathrm{i} \hbar \partial_{\vec{R}_{0}}, \vec{R}_{0}=\sum_{i=1}^{3} \vec{R}_{i} / 3$, represents the 'free' CM kinetic energy, and $V=e^{2}\left(R_{12}^{-1}-R_{13}^{-1}-R_{23}^{-1}\right) \equiv \tilde{V} f$ au.

The magnetic current-current interaction plus the retardation correction corresponding to the lowest order Breit interaction are described by the additional term

$$
\begin{aligned}
M_{2}=-\frac{e^{2}}{2 m^{2} c^{2}} & \left\{R_{12}^{-1}\left[\vec{p}_{1} \cdot \vec{p}_{2}+\hat{\boldsymbol{r}}_{12} \cdot\left(\hat{\boldsymbol{r}}_{12} \cdot \vec{p}_{1}\right) \vec{p}_{2}\right]\right. \\
& \left.-R_{13}^{-1}\left[\vec{p}_{1} \cdot \vec{p}_{3}+\hat{\boldsymbol{r}}_{13} \cdot\left(\hat{\boldsymbol{r}}_{13} \cdot \vec{p}_{1}\right) \vec{p}_{3}\right]-R_{23}^{-1}\left[\vec{p}_{2} \cdot \vec{p}_{3}+\hat{\boldsymbol{r}}_{23} \cdot\left(\hat{\boldsymbol{r}}_{23} \cdot \vec{p}_{2}\right) \vec{p}_{3}\right]\right\}
\end{aligned}
$$

so that the effective Hamiltonian for $\mathrm{Ps}^{-}$which includes the first relativistic corrections is $H=H_{C M}^{0}+H_{0}+H_{1}+H_{2}+M_{2}$.

The operators $\nabla_{k}=a_{\mu} \partial_{\vec{R}_{k}}, \mathrm{k}=1,2,3$, can be expressed in terms of $\nabla_{0}=a_{\mu} \partial_{\vec{R}_{0}}, \nabla_{13}$ and $\nabla_{23}$ by using the relations

$$
\begin{aligned}
& \nabla_{1}=\nabla_{0} / 3+\nabla_{13}, \\
& \nabla_{2}=\nabla_{0} / 3+\nabla_{23}, \\
& \nabla_{3}=\nabla_{0} / 3-\nabla_{13}-\nabla_{23} .
\end{aligned}
$$

Therefore, the term $H_{1}+H_{2}+M_{2}$ can be decomposed in a collective, $\vec{P}_{0}$-dependent part, an intrinsic part and a mixed part, containing both the total momentum $\vec{P}_{0}$ and the intrinsic variables. The mixed terms appear because the internal forces affect the inertial parameter of the whole system, and an exact treatment would provide $E_{D}$ as a function $E_{D}=\sqrt{\left(3 m c^{2}+E_{\mathrm{g}^{*}}\right)^{2}+\left\langle\vec{P}_{0}\right\rangle^{2} c^{2}}$, where $E_{\mathrm{g}^{*}}$ is the ground-state energy given by the pair of equations $H \Psi=E_{\mathrm{g}^{*}} \Psi$ and $\vec{P}_{0}|\Psi\rangle=0$. Thus, even if $H$ cannot be separated in a sum of intrinsic and collective $(\mathrm{CM})$ terms, the translation invariance allows a reduction to the 'dynamical CM frame', described by states in which $\left\langle\vec{P}_{0}\right\rangle=0$. The choice of the Hylleraas basis ensures that $\vec{P}_{0} \Phi=0$ for any variational wavefunction $\Phi$, so that in the expectation values we may take advantage of the reduction simply by changing notation according to the rules:

$$
\begin{aligned}
& \nabla_{1} \rightarrow \nabla_{13}, \\
& \nabla_{2} \rightarrow \nabla_{23}, \\
& \nabla_{3} \rightarrow-\nabla_{13}-\nabla_{23} .
\end{aligned}
$$

The term $H_{1}$ is negative, and can make the energy arbitrarily low with increasing momenta. Therefore, at this level of approximation the system is unstable against collapse around the $\mathrm{CM}$, so that $H$ has no real ground state. In addition, the ground state of $\mathrm{Ps}^{-}$is in fact not stable because of $\mathrm{e}^{+} \mathrm{e}^{-}$annihilation. However, it is possible to define a physical metastable ground state, considering all the correction terms as perturbations with respect to the well-defined, nonrelativistic ground state of $H_{0}$.

The expectation value $\left\langle H_{1}\right\rangle=-(1 / 64)\left\langle\nabla_{1}^{4}+\nabla_{2}^{4}+\nabla_{3}^{4}\right\rangle \alpha^{2} f$ au can be calculated either directly, or by assuming that in the ground state $\left\langle H_{0} \mathcal{O}_{p}\right\rangle=\left\langle\mathcal{O}_{p} H_{0}\right\rangle=E_{\mathrm{g}}\left\langle\mathcal{O}_{p}\right\rangle$ for any operator $\mathcal{O}_{p}$, and using the equalities

$$
\nabla_{13}^{2}+\nabla_{23}^{2}=2\left(\tilde{H}_{0}-\tilde{V}+\nabla_{13} \cdot \nabla_{23} / 2\right)
$$


Table 3. Direct $(\langle *\rangle)$ and $E_{\mathrm{g}}$-dependent $\left(\langle *\rangle_{E}\right)$ expectation values of the singular operators $\nabla_{1}^{4}$ and $\nabla_{3}^{4}$ as a function of the basis dimension $N_{\mathrm{b}}$.

\begin{tabular}{llll}
\hline$N_{\mathrm{b}}$ & $\left\langle\nabla_{1}^{4}\right\rangle$ & $\left\langle\nabla_{1}^{4}\right\rangle_{E}$ & $\left\langle\nabla_{3}^{4}\right\rangle_{E}$ \\
\hline 324 & 2.53244571929 & 2.5324510044426 & 5.255396862891 \\
411 & 2.53245169756 & 2.5324510504206 & 5.255397122254 \\
512 & 2.53245074184 & 2.5324510568770 & 5.255397117353 \\
630 & 2.53244996461 & 2.5324510091320 & 5.255397051034 \\
764 & 2.53245099221 & 2.5324510187191 & 5.255397086467 \\
918 & 2.53245105652 & 2.5324510224536 & 5.255397094127 \\
1089 & 2.53245102343 & 2.5324510215297 & 5.255397091672 \\
1283 & 2.53245101949 & 2.5324510205893 & 5.255397091024 \\
1495 & 2.53245102217 & 2.5324510205950 & 5.255397090993 \\
1733 & 2.53245102024 & 2.5324510205872 & 5.255397090958 \\
1990 & 2.53245101992 & 2.5324510205592 & 5.255397090940 \\
2276 & 2.53245102043 & 2.5324510205596 & 5.255397090949 \\
2528 & 2.53245102042 & 2.5324510205600 & 5.255397090945 \\
Extrap. & $2.53245102,2(2)$ & $2.5324510205596(3)$ & $5.255397090945(4)$ \\
\hline
\end{tabular}

with $\tilde{H}_{0}=H_{0} /(f$ au $)$, and

$\nabla_{13}^{4}+\nabla_{23}^{4}=4\left(\tilde{H}_{0}-\tilde{V}+\nabla_{13} \cdot \nabla_{23} / 2\right)^{2}-2 \nabla_{13}^{2} \nabla_{23}^{2}$,

$\left\langle\nabla_{3}^{4}\right\rangle=\left\langle\nabla_{13}^{4}+\nabla_{23}^{4}+4\left(\nabla_{13} \cdot \nabla_{23}\right)^{2}\right\rangle+\left\langle 2 \nabla_{13}^{2} \nabla_{23}^{2}+4\left(\nabla_{13}^{2}+\nabla_{23}^{2}\right) \nabla_{13} \cdot \nabla_{23}\right\rangle$.

Although formally the same, within a finite basis the two evaluations (direct and energy dependent) give slightly different results $\left(\left\langle\nabla^{4}\right\rangle,\left\langle\nabla^{4}\right\rangle_{E}\right)$ as shown in table 3, and further discussed in appendix A. To estimate $\left\langle H_{1}\right\rangle$ we have used only $\left\langle\nabla^{4}\right\rangle_{E}$, because of its more rapid convergence and higher accuracy in the extrapolated value.

The term $H_{2}$ contains the singular operators $\nabla_{1}^{2} \tilde{V}=-4 \pi\left[\delta\left(\vec{r}_{12}\right)-\delta\left(\vec{r}_{13}\right)\right], \nabla_{2}^{2} \tilde{V}=$ $-4 \pi\left[\delta\left(\vec{r}_{12}\right)-\delta\left(\vec{r}_{23}\right)\right]$ and $\nabla_{3}^{2} \tilde{V}=4 \pi\left[\delta\left(\vec{r}_{13}\right)+\delta\left(\vec{r}_{23}\right)\right]$, which yield

$$
\left\langle H_{2}\right\rangle=\alpha^{2} \pi\left\langle\delta\left(\vec{r}_{13}\right)+\delta\left(\vec{r}_{23}\right)-\delta\left(\vec{r}_{12}\right)\right\rangle f^{3} \text { au. }
$$

Previous estimates of $\left\langle\delta\left(\vec{R}_{13}\right)\right\rangle \quad\left(=a_{\mu}^{-3}\left\langle\delta\left(\vec{r}_{13}\right)\right\rangle\right)$ in $\mathrm{Ps}^{-}$by using the correlationfunction hyperspherical-harmonic method and the stochastic variational method are $0.02073314(6) a_{0}^{-3}$, respectively $0.020731048976 a_{0}^{-3}$ [14]. The same methods give for $\left\langle\delta\left(\vec{R}_{12}\right)\right\rangle$ the values $0.000170997(2) a_{0}^{-3}$ and $0.000171112600741 a_{0}^{-3}$, respectively [14]. The results of the present calculation, in the same units $\left(a_{0}^{-3}\right)$, are listed in table 4 , as a function of the dimension $N_{\mathrm{b}}$ of the basis set. The error estimates for all the results due to the convergence of the basis set are discussed in appendix B.

The expectation values which appear in the calculation of $\left\langle M_{2}\right\rangle$, obtained when $N_{\mathrm{b}}=324$ are

$$
\begin{aligned}
& u_{\mathrm{ee}}=\left\langle r_{12}^{-1} \nabla_{1} \cdot \nabla_{2}\right\rangle=-0.00826764667, \\
& v_{\mathrm{ee}}=\left\langle r_{12}^{-1} \hat{r}_{12} \cdot\left(\hat{r}_{12} \cdot \nabla_{1}\right) \nabla_{2}\right\rangle=0.01961092535
\end{aligned}
$$

and for $i=1,2$

$$
\begin{aligned}
& u_{\text {ep }}=\left\langle r_{i 3}^{-1} \nabla_{i} \cdot \nabla_{3}\right\rangle=1.53543404931 \\
& v_{\text {ep }}=\left\langle r_{i 3}^{-1} \hat{r}_{i 3} \cdot\left(\hat{r}_{i 3} \cdot \nabla_{3}\right) \nabla_{i}\right\rangle=-0.555009821912 .
\end{aligned}
$$


Table 4. Ground-state expectation values of the singular distributions $\delta\left(\vec{R}_{13}\right)$ and $\delta\left(\vec{R}_{12}\right)$ as a function of the basis dimension $N_{\mathrm{b}}$.

\begin{tabular}{lll}
\hline$N_{\mathrm{b}}$ & $\left\langle\delta\left(\vec{R}_{13}\right)\right\rangle\left[a_{0}^{-3}\right]$ & $\left\langle\delta\left(\vec{R}_{12}\right)\right\rangle\left[a_{0}^{-3}\right]$ \\
\hline 324 & 0.0207331742302 & 0.0001710000008 \\
411 & 0.0207332038381 & 0.0001709993832 \\
512 & 0.0207331998045 & 0.0001709999672 \\
630 & 0.0207331932922 & 0.0001709973067 \\
764 & 0.0207331979867 & 0.0001709968854 \\
918 & 0.0207331982389 & 0.0001709968110 \\
1089 & 0.0207331980943 & 0.0001709968324 \\
1283 & 0.0207331979995 & 0.0001709967560 \\
1495 & 0.0207331980243 & 0.0001709967673 \\
1733 & 0.0207331980074 & 0.0001709967601 \\
1990 & 0.0207331980034 & 0.0001709967577 \\
2276 & 0.0207331980053 & 0.0001709967571 \\
2528 & 0.0207331980050 & 0.0001709967568 \\
Extrap. & $0.0207331980046(8)$ & $0.0001709967567(4)$ \\
\hline
\end{tabular}

(The sum $u_{\text {ep }}+v_{\text {ep }}=0.980424227$ corresponds to the quantity $1-J$, where $J$ is the contribution to the $\mathrm{Ps}^{-}$binding energy tabulated by Bhatia and Drachman [20].) In terms of these variables, $\left\langle M_{2}\right\rangle=0.5 \alpha^{2} w f^{3}$ au with $w=u_{\text {ee }}+v_{\text {ee }}-2 u_{\text {ep }}-2 v_{\text {ep }}=-1.949505176125$. For the 2528-dimensional basis set $w=-1.949505250368$. The average of the last three consecutive values, obtained for $N_{\mathrm{b}}=1990,2276$ and 2528, gives the matrix element $w=-1.949505250368(1)$.

The sum of the spin-independent relativistic corrections $\left\langle H_{1}\right\rangle+\left\langle H_{2}\right\rangle+\left\langle M_{2}\right\rangle$ is $-0.145476184397(8) \alpha^{2} f$ au, which decreases the $\mathrm{Ps}^{-}$ground-state energy to

$$
E_{\mathrm{g}^{*}}=\langle H\rangle=E_{\mathrm{g}}-0.145476184397(8) \alpha^{2} f \mathrm{au} .
$$

For comparison, the same calculations yield for the corrected ground-state energy $E_{\mathrm{g}^{*}}^{0}$ of neutral positronium $E_{\mathrm{g}^{*}}^{0}=-\left(0.5+5 \alpha^{2} / 32\right) f$ au. This result can also be obtained by using the expansion $E_{(1,1 / 2,1)} \approx-\left(0.5+5 \alpha^{2} / 32\right) f$ au of the energy $E_{(n, j, Z)}$ provided by the one-body Dirac equation [24]

$$
E_{(n, j, Z)}=\frac{1}{\alpha^{2}}\left[\eta-1-\frac{\mu}{2\left(m+m_{3}\right)}(\eta-1)^{2}\right] f \mathrm{au},
$$

where $\eta=1 / \sqrt{1+(Z \alpha)^{2} /(n-v)^{2}}, v=j+1 / 2-\sqrt{(j+1 / 2)^{2}-(Z \alpha)^{2}}$. The numerical values presented in this section can be compared with the previous results available in the literature [20]. Thus, the term $\left\langle H_{1}\right\rangle$ of table 6 is close to the value $-0.16124946 \alpha^{2} \mathrm{Ry}$ obtained in [20], but for the other two terms we have found significant differences.

\section{QED corrections}

Within QED the constituents of the three-body system $\mathrm{e}^{-} \mathrm{e}^{+} \mathrm{e}^{-}$cease to be 'elementary', because they are subject not only to the mutual two-body Coulomb-Breit interaction, but are also coupled to the vacuum fluctuations of the electromagnetic field $\vec{A}$ [25]. The interaction terms accounting for this coupling are represented by an infinite series of increasingly complicated Feynman diagrams with closed photon lines. However, the complexity increases recursively, by taking into account at each order three basic processes, represented by the 
anomalous magnetic moment (vertex) corrections, electron self-mass and vacuum polarization diagrams.

Although formally complicated, the main effect of the coupling to the field degrees of freedom is simply a change in the charge and mass parameters $e$ and $m$ of the theory. This contribution has already been taken into account, because it is included in the measured values of $e$ and $m$ used to define the atomic unit of energy. Though, the QED corrections in the interacting three-body system $\mathrm{e}^{-} \mathrm{e}^{+} \mathrm{e}^{-}$are not the same as for the free particles, and the differences still need to be considered.

The vacuum polarization properties have been studied first by Heisenberg [26] and Uehling [27], showing that a given charge density $\rho(\vec{R})$ induces a polarization charge $\delta \rho(\vec{R})=-(\alpha / 15 \pi) \lambda_{0}^{2} \Delta_{R} \rho(\vec{R})$, where $\lambda_{0}=\hbar / m c$ is the Compton wavelength of the electron and $\Delta_{R} \equiv \partial_{\vec{R}}^{2}$. The induced charge leads to deviations from the standard Coulomb interaction. Thus, the vacuum behaves as an inhomogeneous dielectric, in which the mutual potential energy between two point-like charges $Z_{1}$ and $Z_{2}$ is [27]

$$
V(R)=\frac{Z_{1} Z_{2} e^{2}}{R}\left[1-\frac{\alpha}{\pi} R U(R)\right],
$$

where $U(R)$ denotes the Uehling potential. This potential is singular at $R=0$, falls of exponentially for $R>0$ and satisfies the integral condition $\int \mathrm{d}^{3} R U(R)=-4 \pi \lambda_{0}^{2} / 15$. Therefore, it can be well approximated by a delta function, $U(R)=-4 \pi\left(\lambda_{0}^{2} / 15\right) \delta(\vec{R})$. In the case of $\mathrm{Ps}^{-}$, the correction introduced by this potential is

$$
\left\langle H_{\mathrm{vp}}\right\rangle=\frac{4}{15} \alpha^{3}\left\langle\delta\left(\vec{r}_{12}\right)-\delta\left(\vec{r}_{13}\right)-\delta\left(\vec{r}_{23}\right)\right\rangle f^{3} \mathrm{au}
$$

Using the expectation values given in table 4 , the contribution of the vacuum polarization to the $\mathrm{Ps}^{-}$ground-state energy is $\left\langle H_{\mathrm{vp}}\right\rangle=-0.0220242129346(7) \alpha^{3} f$ au. It is important to remark that this value takes into account the positron recoil (the 'mass polarization' term) because the wavefunctions are obtained by minimizing the full nonrelativistic Hamiltonian. In neutral positronium $\left\langle\delta\left(\vec{R}_{13}\right)\right\rangle_{\mathrm{Ps}}=1 /\left(\pi a_{\mu}^{3}\right)=1 /\left(8 \pi a_{0}^{3}\right)$, and the vacuum polarization correction is $-1 /(15 \pi) \alpha^{3} f$ au.

As was shown early by the Lamb shift measurements [28], the main QED correction comes from the coupling to the vacuum fluctuations of the field rather than from the vacuum polarization ([25] p 59). For a free electron the relativistic ground state energy is given by its rest mass $m=m_{b}+\delta m$, consisting of the uncoupled value $m_{b}$ and the positive renormalization constant $\delta m=\left(3 \alpha m_{b} / 2 \pi\right) \ln \left(\Lambda / m_{b}\right)$ due to the electromagnetic self-energy, where $\Lambda$ is a large (formally infinite) cut-off mass.

Similarly, the coupling to the field modes also affects the intrinsic excitations of a manybody system. In a bound $N$-particle system, the shift $\Delta E_{n}$ in the energy $E_{n}=\left\langle n\left|H_{0}\right| n\right\rangle$ of the level $|n\rangle$ due to the exchange of a transverse photon can be obtained by using the time-independent second-order perturbation expression

$$
\Delta E_{n}=-\left\langle n, 0_{f}\left|H_{c}(\vec{A}) \frac{1}{H_{0}+H_{A}-E_{n}} H_{c}(\vec{A})\right| n, 0_{f}\right\rangle .
$$

Here $H_{c}(\vec{A})=\sum_{i=1}^{N} h_{i}(\vec{A})$ is the sum over all particles of the one-body coupling terms $h_{i}(\vec{A})=-e_{i} \vec{p}_{i} \cdot \vec{A}_{\left(\vec{R}_{i}\right)} / m_{i} c$, where

$$
\vec{A}_{(\vec{r})}=\frac{\sqrt{\hbar c}}{2 \pi} \int \frac{\mathrm{d}^{3} k}{\sqrt{k}} \sum_{\lambda=1,2} \vec{\epsilon}_{\lambda}\left(\hat{\boldsymbol{a}}_{k \lambda}^{\dagger} \mathrm{e}^{-\mathrm{i} \vec{k} \cdot \vec{r}}+\hat{\boldsymbol{a}}_{k \lambda} \mathrm{e}^{\mathrm{i} \vec{k} \cdot \vec{r}}\right)
$$


is the quantized transverse vector potential of the photon $\left(\vec{\epsilon}_{\lambda} \cdot \vec{k}=0, \vec{\epsilon}_{\lambda}^{2}=1\right), H_{A}=$ $\int \mathrm{d}^{3} k \sum_{\lambda=1,2} \hbar c k a_{k \lambda}^{\dagger} a_{k \lambda}$ is the free field Hamiltonian and $\left|0_{f}\right\rangle$ denotes the photon vacuum. This shift has the form $\Delta E_{n}=\sum_{i=1}^{N} X_{i}^{n}+\sum_{i<j} Y_{i j}^{n}$, where

$$
X_{i}^{n}=-\left\langle n, 0_{f}\left|h_{i}(\vec{A}) \frac{1}{H_{0}+H_{A}-E_{n}} h_{i}(\vec{A})\right| n, 0_{f}\right\rangle
$$

and

$$
Y_{i j}^{n}=-2 \operatorname{Re}\left[\left\langle n, 0_{f}\left|h_{i}(\vec{A}) \frac{1}{H_{0}+H_{A}-E_{n}} h_{j}(\vec{A})\right| n, 0_{f}\right\rangle\right] .
$$

It is important to remark that the interaction with the vacuum field fluctuations may affect not only the intrinsic dynamics, but also the centre of mass. In a classical two-body system coupled to the field, $H_{c}$ can be written in terms of the canonical pairs $\left(\vec{R}_{12}, \vec{p}_{12}\right) \equiv$ $\left(\vec{R}_{1}-\vec{R}_{2}, \mu \vec{p}_{1} / m_{1}-\mu \vec{p}_{2} / m_{2}\right)$ and $\left(\vec{R}_{0}, \vec{P}_{0}\right) \equiv\left(\mu \vec{R}_{1} / m_{2}+\mu \vec{R}_{2} / m_{1}, \vec{p}_{1}+\vec{p}_{2}\right)$ of intrinsic and, respectively, $\mathrm{CM}$ variables as

$$
\vec{p}_{12} \cdot\left[\frac{e_{2}}{m_{2}} \vec{A}_{\left(R_{2}\right)}-\frac{e_{1}}{m_{1}} \vec{A}_{\left(R_{1}\right)}\right]-\frac{\mu}{m_{1} m_{2}} \vec{P}_{0} \cdot\left[e_{1} \vec{A}_{\left(R_{1}\right)}+e_{2} \vec{A}_{\left(R_{2}\right)}\right] .
$$

This expression shows that in a neutral two-body system (such as Ps), the CM energy is not affected by the field only if $\vec{A}_{\left(R_{1}\right)}=\vec{A}_{\left(R_{2}\right)}$, or when the size of the system is negligible compared to the photon wavelength (dipole approximation).

In a quantum $N$-body system it is convenient to take advantage of the finite size effects by writing $\vec{A}$ as an incoherent sum of long and short wavelength components, $\vec{A}_{\mathrm{L}}$ and $\vec{A}_{\mathrm{S}}$, obtained by decomposing $\int \mathrm{d}^{3} k$ as $\int_{|\vec{k}| \leqslant k_{L}} \mathrm{~d}^{3} k+\int_{k_{L}<|\vec{k}|<k_{M}} \mathrm{~d}^{3} k$, where $k_{L}$ and $k_{M}$ are cut-off parameters. Each domain brings its own contribution to the matrix elements, which can be similarly decomposed as

$$
X_{i}^{n}=X_{i}^{L n}+X_{i}^{S n}, \quad Y_{i j}^{n}=Y_{i j}^{L n}+Y_{i j}^{S n} .
$$

At the end of the calculation $k_{L}$ should disappear, while $k_{M} \rightarrow \infty$.

If $H_{0}$ consists of the kinetic energy term plus a local potential $V$, then a nonrelativistic calculation within the dipole approximation yields

$$
X_{i}^{L n}=-\frac{\alpha}{3 \pi m^{2} c^{2}}\left[2 \hbar c k_{L}\left\langle n\left|p_{i}^{2}\right| n\right\rangle+\left\langle n\left|\left[\vec{p}_{i} \cdot,\left[\vec{p}_{i}, V\right]\right]\right| n\right\rangle \ln \frac{k_{L}}{k_{R}}-2 B_{i i}^{n}\right],
$$

where $k_{R}=R_{M} / \hbar c, R_{M}$ is a dimensional constant with units of energy and $B_{i i}^{n}$ are the diagonal elements of the matrix $\left[B_{i j}^{n}\right]$ defined by

$$
B_{i j}^{n}=\sum_{m}\left(E_{n}-E_{m}\right) \operatorname{Re}\left(\left\langle n\left|\vec{p}_{i}\right| m\right\rangle \cdot\left\langle m\left|\vec{p}_{j}\right| n\right\rangle\right) \ln \frac{\left|E_{n}-E_{m}\right|}{R_{M}} .
$$

The first term in equation (43) depends only on the kinetic energy, and it can be written as $-\delta m_{L}\left\langle p_{i}^{2}\right\rangle_{n} / 2 m^{2}, \delta m_{L}=4 m r_{e} k_{L} / 3 \pi$, where $r_{e}=\alpha \hbar / m c$ denotes the classical radius of the electron. It contributes also to the energy of a free particle $(V=0)$ and has the structure of a first-order perturbation shift induced by a variation $\delta m_{L}$ of the nonrelativistic mass. Thus, such terms can be taken into account simply by a redefinition of the cut-off mass $\Lambda$.

A relativistic calculation of the one-body QED correction arising from the exchange of a transverse hard photon at a Coulomb vertex $[29,25] \mathrm{p} 177$, yields

$$
X_{i}^{S n}=\frac{\alpha \hbar^{2}}{3 \pi m^{2} c^{2}}\left(\ln \frac{m c}{2 \hbar k_{L}}+\frac{5}{6}\right)\left\langle n\left|\Delta_{R_{i}} V\right| n\right\rangle,
$$


$(5 / 6=11 / 24+3 / 8)$ so that

$X_{i}^{n}=-\frac{\delta m_{L}}{2 m^{2}}\left\langle p_{i}^{2}\right\rangle_{n}+\frac{\alpha}{3 \pi m^{2} c^{2}}\left[\hbar^{2}\left(\ln \frac{m c}{2 \hbar k_{R}}+\frac{5}{6}\right)\left\langle\Delta_{R_{i}} V\right\rangle_{n}+2 B_{i i}^{n}\right]$.

The quantity $Y_{i j}^{L n}$ can be expressed as

$Y_{i j}^{L n}=-\frac{\delta m_{L}}{m^{2}} \frac{e_{i} e_{j}}{e^{2}}\left\langle\vec{p}_{i} \cdot \vec{p}_{j}\right\rangle_{n}-\frac{2 \alpha}{3 \pi m^{2} c^{2}} \frac{e_{i} e_{j}}{e^{2}}\left\{\left\langle\left[\vec{p}_{i} \cdot,\left[\vec{p}_{j}, V\right]\right]\right\rangle_{n} \ln \frac{k_{L}}{k_{R}}-2 B_{i j}^{n}\right\}$.

In the case of $\mathrm{Ps}^{-}$there are three matrix elements $X_{i}^{n}$, two for the electrons $(i=1,2)$ and one for the positron $(i=3)$, and three $Y_{i j}^{L n}, i<j$. The contribution to $\Delta E_{n}$ arising from the part linear in $\delta m_{L}$ of $X$ and $Y^{L}$ is $\delta_{0} E_{n}=-\delta m_{L}\left\langle\left(\vec{p}_{1}+\vec{p}_{2}-\vec{p}_{3}\right)^{2}\right\rangle_{n} / 2 m^{2}$. In the dynamical CM frame, this energy shift can be accounted, for example, by an effective variation $3 \delta m_{L}$ in the total mass of the electron-electron pair and $\delta m_{L}$ in the mass of the positron, or by a variation of $4 \delta m_{L}$ in only one of them.

The definition of the Bethe logarithm $\beta_{n} \equiv 2 B_{33}^{n} /\left\langle\left[\vec{p}_{3}, \cdot\left[\vec{p}_{3}, H_{0}\right]\right]\right\rangle_{n}$, and the identity $m^{2} \sum_{i, j} e_{i} e_{j} B_{i j}^{n} /\left(m_{i} m_{j}\right)=e^{2} B_{33}^{n}\left(1+m / m_{3}\right)^{2}$ (valid if $m_{1}=m_{2}=m$ and $\left\langle n\left|\vec{P}_{0}\right| n^{\prime}\right\rangle=0$ for any $\left.n, n^{\prime}\right)$ show that $\sum_{i} X_{i}^{n}+\sum_{i<j} Y_{i j}^{L n}=\delta_{0} E_{n}+\delta_{1} E_{n}+\delta_{2 L} E_{n}$, where

$$
\begin{aligned}
\delta_{1} E_{n} & =\frac{\alpha \hbar^{2}}{3 \pi m^{2} c^{2}}\left[-4 \beta_{n}\left\langle\Delta_{R_{3}} V\right\rangle_{n}+\left(\ln \frac{m c}{2 \hbar k_{R}}+\frac{5}{6}\right) \sum_{i=1}^{3}\left\langle\Delta_{R_{i}} V\right\rangle_{n}\right] \\
& =\frac{4 \alpha^{3}}{3}\left[-4 \beta_{n}\left\langle\delta\left(\vec{r}_{13}\right)+\delta\left(\vec{r}_{23}\right)\right\rangle_{n}+2\left(\ln \frac{m c}{2 \hbar k_{R}}+\frac{5}{6}\right)\left\langle\delta\left(\vec{r}_{13}\right)+\delta\left(\vec{r}_{23}\right)-\delta\left(\vec{r}_{12}\right)\right\rangle_{n}\right] f^{3} \mathrm{au}
\end{aligned}
$$

and $\delta_{2 L} E_{n} \equiv \sum_{i<j}\left\langle W_{i j}^{L}\right\rangle_{n}$ is given by the expectation value of the potential

$$
W_{i j}^{L}\left(k_{L}\right)=\frac{8 \alpha^{3}}{3} \ln \frac{k_{L}}{k_{R}} \delta\left(\vec{r}_{i j}\right) f^{3} \text { au. }
$$

The element $Y_{i j}^{S n}$ due to the exchange of a short wavelength (hard) transverse photon between different particles will be decomposed as $Y_{i j}^{S n}=Y_{i j}^{S 2 n}+Y_{i j}^{S 3 n}$, according to the expansion $1 /\left(H_{0}+H_{A}-E_{n}\right) \approx 1 / H_{A}-\left(H_{0}-E_{n}\right) /\left(H_{A}\right)^{2}$. The contribution from $1 / H_{A}$ is

$$
Y_{i j}^{S 2 n}=-2\left\langle n, 0_{f}\left|h_{i}\left(\vec{A}_{S}\right) H_{A}^{-1} h_{j}\left(\vec{A}_{S}\right)\right| n, 0_{f}\right\rangle .
$$

In the limit $k_{L} \rightarrow 0, k_{M} \rightarrow \infty$, the integral over $k$ in this matrix element can be evaluated by using the identity

$$
\int \frac{\mathrm{d}^{3} k}{k^{2}} \mathrm{e}^{\mathrm{i} \vec{k} \cdot \vec{r}}(\vec{A} \cdot \vec{B}-\hat{\boldsymbol{k}} \cdot \vec{A} \hat{\boldsymbol{k}} \cdot \vec{B})=\frac{\pi}{r}(\vec{A} \cdot \vec{B}+\hat{\boldsymbol{r}} \cdot \vec{A} \hat{\boldsymbol{r}} \cdot \vec{B}),
$$

showing that the sum $\sum_{i<j} Y_{i j}^{S 2 n}$ becomes the two-body correction $\left\langle M_{2}\right\rangle_{n}$ of order $\alpha^{2} \mathrm{Ry}$, already taken into account. Thus, only the next term brings a new contribution,

$$
Y_{i j}^{S 3 n}=2 \operatorname{Re}\left[\left\langle n, 0_{f}\left|h_{i}\left(\vec{A}_{S}\right) \frac{H_{0}-E_{n}}{H_{A}^{2}} h_{j}\left(\vec{A}_{S}\right)\right| n, 0_{f}\right\rangle\right]
$$

which is the expectation value of the two-body potential

$$
W_{i j}^{S}\left(k_{L}, k_{M}\right)=\frac{2 \alpha^{3}}{3 \pi}\left[\frac{3}{2} F\left(k_{L}, k_{M}, r_{i j}\right)+4 \pi \delta\left(\vec{r}_{i j}\right) \ln \frac{k_{M}}{k_{L}}\right] f^{3} \text { au. }
$$

Here $F\left(k_{L}, k_{M}, r\right)=2\left[j_{0}\left(k_{M} r\right)+j_{2}\left(k_{M} r\right)-j_{0}\left(k_{L} r\right)-j_{2}\left(k_{L} r\right)\right] /\left(3 r^{3}\right)$ is the function introduced by Araki [30], written in terms of the spherical Bessel functions $j_{0}, j_{2}$. When 
$k_{L} \rightarrow 0, k_{M} \rightarrow \infty, F(0, \infty, r)=-2 /\left(3 r^{3}\right)$, but the logarithmic factor in the second term of $W_{i j}^{S}$ is divergent at both limits. However, the divergence in $k_{L}$ is cancelled by the low-energy term, and the sum $W_{i j}\left(k_{M}\right)=W_{i j}^{L}\left(k_{L}\right)+W_{i j}^{S}\left(k_{L}, k_{M}\right)$,

$$
W_{i j}\left(k_{M}\right)=\frac{2 \alpha^{3}}{3 \pi}\left[\frac{3}{2} F\left(0, k_{M}, r_{i j}\right)+\frac{\delta\left(r_{i j}\right)}{r_{i j}^{2}} \ln \frac{k_{M}}{k_{R}}\right] f^{3} \mathrm{au},
$$

is independent of $k_{L}$. The divergent factor containing $k_{M}$ contributes only when $|n\rangle$ is an $S$ state, but in this case the expectation value $\left\langle 1 / r^{3}\right\rangle_{n}$ is also logarithmically divergent. It is, however, possible to define a limit for the sum of these infinite quantities in the sense of the principal value. Let

$$
D(a, r)=\frac{\theta(r-a)}{r^{3}}-\frac{\delta(r)}{r^{2}} \ln \frac{a_{\mu}}{a}
$$

be a distribution depending on the positive radius parameter $a=\eta / k_{M}$, where $\eta$ is a positive scale factor. Because $r^{2} \partial_{a} D(a, r)=[\delta(r)-\delta(r-a)] / a$, when $k_{M} \rightarrow \infty$ the expectation value $\langle D(a, r)\rangle_{n}$ is finite. In terms of this distribution we can define the principal value

$$
\left.\mathcal{P}\left[\frac{3}{2} F\left(0, k_{M}, r\right)+\frac{\delta(r)}{r^{2}} \ln \frac{k_{M}}{k_{R}}\right]\right|_{k_{M} \rightarrow \infty}=4 \pi \delta(\vec{r}) \ln \frac{\eta}{a_{\mu} k_{R}}-\lim _{a \rightarrow 0} D(a, r) .
$$

The choice of a scale factor $\eta=\mathrm{e}^{\frac{4}{3}-\gamma}$, where $\gamma$ is the Euler's constant, leads to the formula used by Araki [30]

$$
\left\langle W_{i j}\right\rangle_{n}=-\frac{2 \alpha^{3}}{3 \pi}\left[Q_{i j}^{n}+4 \pi\left\langle\delta\left(\vec{r}_{i j}\right)\right\rangle_{n}\left(\ln a_{\mu} k_{R}-\frac{4}{3}\right)\right] f^{3} \mathrm{au},
$$

where $Q_{i j}^{n}=\lim _{a \rightarrow 0}\left\langle D(a, r)+4 \pi \gamma \delta\left(\vec{r}_{i j}\right)\right\rangle_{n}$. In Ps ${ }^{-}$this gives for the effective two-body contribution $\delta_{2} E_{n}=\delta_{2 L} E_{n}+\sum_{i<j}\left\langle W_{i j}^{S}\right\rangle_{n}=\sum_{i<j}\left\langle W_{i j}\right\rangle_{n}$ the expression

$$
\delta_{2} E_{n}=-\frac{2 \alpha^{3}}{3 \pi}\left[Q_{12}^{n}+Q_{13}^{n}+Q_{23}^{n}+4 \pi\left(\ln a_{\mu} k_{R}-\frac{4}{3}\right)\left\langle n\left|\delta\left(\vec{r}_{13}\right)+\delta\left(\vec{r}_{23}\right)+\delta\left(\vec{r}_{12}\right)\right| n\right\rangle\right] f^{3} \text { au. }
$$

According to these partial results, the effective QED contribution of order $\alpha^{3}$ to the energy level $E_{n}$ of $\mathrm{Ps}^{-}$due to the exchange of a transverse photon is $\delta_{1 p} E_{n}=\delta_{1} E_{n}+\delta_{2} E_{n}$. This sum is independent of the arbitrary energy unit $R_{M}$, as it should be, but to simplify the numerical calculations we choose $R_{M}=f$ Ry. With this choice, $a_{\mu} k_{R}=\alpha / 2$, and $m c /\left(2 \hbar k_{R}\right)=1 /\left(f \alpha^{2}\right)$.

The corrections in neutral positronium can be obtained from the expressions given above simply by neglecting all the expectation values containing the variables $r_{23}$ and $r_{12}$, involving the second electron. For the Ps ground state $Q_{13}^{g 0}=-4 \ln 2$, while $\beta_{g}^{0}$ is the same as the Bethe logarithm for hydrogen, $\beta_{g}^{H}=2.984128555765497611(4)$, each logarithm being calculated using the corresponding reduced Rydberg constant [31]. In the case of $\mathrm{Ps}^{-}$the numerical values $Q_{13}^{g}$ and $Q_{12}^{g}$ used in the present estimates are listed in table 5, while $\beta_{g}=3.005030(2)$ [31] (including the finite mass correction).

To the same order we should also consider the contribution $\delta_{2 p} E_{n}[30,32]$ of the twophoton exchange term (including the Coulomb part),

$$
\begin{aligned}
\delta_{2 p} E_{n}=-\frac{\alpha^{3}}{2 \pi} & {\left[Q_{12}^{n}+Q_{13}^{n}+Q_{23}^{n}-4 \pi\left(\ln f \alpha-\frac{4}{3} \ln 2+\frac{13}{6}\right)\right.} \\
& \left.\times\left\langle n\left|\delta\left(\vec{r}_{13}\right)+\delta\left(\vec{r}_{23}\right)+\delta\left(\vec{r}_{12}\right)\right| n\right\rangle\right] f^{3} \mathrm{au},
\end{aligned}
$$

and the energy shift associated with two-photon decay. 
Table 5. The ground-state expectation values $Q_{1 k}^{g}=\lim _{a \rightarrow 0}\left\langle\theta\left(r_{1 k}-a\right) / r_{1 k}^{3}+4 \pi[\gamma+\right.$ $\left.\left.\ln \left(a / a_{\mu}\right)\right] \delta\left(\vec{r}_{1 k}\right)\right\rangle$ as a function of the basis dimension $N_{\mathrm{b}}$.

\begin{tabular}{lll}
\hline$N_{\mathrm{b}}$ & $Q_{13}^{g}\left[a_{\mu}^{-3}\right]$ & $Q_{12}^{g}\left[a_{\mu}^{-3}\right]$ \\
\hline 324 & -2.7765632953 & 0.09575778075 \\
411 & -2.7765883435 & 0.09575797579 \\
512 & -2.7765838294 & 0.09575780427 \\
630 & -2.7765786875 & 0.09575874978 \\
764 & -2.7765828102 & 0.09575890403 \\
918 & -2.7765828944 & 0.09575893040 \\
1089 & -2.7765827757 & 0.09575891840 \\
1283 & -2.7765826945 & 0.09575894978 \\
1495 & -2.7765827219 & 0.09575894476 \\
1733 & -2.7765827034 & 0.09575894786 \\
1990 & -2.7765827003 & 0.09575894904 \\
2276 & -2.7765827022 & 0.09575894931 \\
2528 & -2.7765827019 & 0.09575894943 \\
Extrap. & $-2.776582702(1)$ & $0.0957589494(1)$ \\
\hline
\end{tabular}

A useful check that we obtain the known QED correction for helium (or anti-helium) in the limit where the mass of the positive charge becomes large is discussed in appendix $\mathrm{C}$.

In general, any coupling which makes the levels unstable produces a complex energy shift $\delta_{c} E_{n}-\mathrm{i} \Gamma_{c}^{n} / 2$, where $\delta_{c} E_{n}$ is a correction to the level centroid, $\lambda_{c}^{n}=\Gamma_{c}^{n} / \hbar$ is the decay rate and $c$ denotes the decay channel. Neutral positronium normally decays by spontaneous $\mathrm{e}^{+} \mathrm{e}^{-}$annihilation into two photons if the total spin $S_{13}=0,\left(\vec{S}_{i j}=\vec{s}_{i}+\vec{s}_{j}\right)$, and in three photons if $S_{13}=1$ [33]. The corresponding decay rates are so that $\Gamma_{(\mathrm{Ps}, 3 \gamma)}^{n} \sim \alpha \Gamma_{(\mathrm{Ps}, 2 \gamma)}^{n}$, and the first correction arises from the two-photon annihilation. In this channel $\delta_{2 \gamma} E_{n} / \Gamma_{(\mathrm{Ps}, 2 \gamma)}^{n}=-(1-\ln 2) / \pi$ [34], where

$$
\Gamma_{(\mathrm{Ps}, 2 \gamma)}^{n}=2 \pi \alpha^{3}\left\langle\left(2-\vec{S}_{13}^{2}\right) \delta\left(\vec{r}_{13}\right)\right\rangle_{n} f^{3} \text { au. }
$$

For the Ps ground state $\left(S_{13}=0\right)$ this gives a decay rate $\lambda_{(\mathrm{Ps}, 2 \gamma)}=\alpha^{3} \mathrm{Ry} / \hbar=8.04 \mathrm{~ns}^{-1}$, close to the experimental result $7.99(11) \mathrm{ns}^{-1}$ [35].

In the $\mathrm{Ps}^{-}$ground state the electron spins are coupled to 0 , and the two-photon annihilation can take place between the positron and either of the two electrons. The total rate depends on $\left\langle\vec{S}_{13}^{2}+\vec{S}_{23}^{2}\right\rangle=3$, and can be expressed in the form

$$
\Gamma_{\left(\mathrm{Ps}^{-}, 2 \gamma\right)}=2 \pi \alpha^{3}\left\langle\delta\left(\vec{r}_{13}\right)\right\rangle f^{3} \text { au. }
$$

The ground-state expectation value $\left\langle\delta\left(\vec{R}_{13} / a_{0}\right)\right\rangle$ given in table 4 yields $\lambda_{\left(\mathrm{Ps}^{-}, 2 \gamma\right)}=$ 2.092 797(1) $\mathrm{ns}^{-1}$, in good agreement with the previous estimates [12] and the experimental result 2.09(9) $\mathrm{ns}^{-1}$ [2]. Assuming the same ratio $\delta_{2 \gamma} E / \Gamma_{2 \gamma}$ as in the case of positronium, the corresponding level shift is $\delta_{2 \gamma} E_{\mathrm{g}}=-2 \alpha^{3}(1-\ln 2)\left\langle\delta\left(\vec{r}_{13}\right)\right\rangle f^{3}$ au.

Summarizing the results of the present calculations, the effective ground-state expectation values of the first relativistic and QED correction terms for $\mathrm{Ps}$ and $\mathrm{Ps}^{-}$are collected in table 6 .

In $\mathrm{Ps}^{-}$the nonrelativistic one-electron binding energy $B_{1 \mathrm{e}}=-1 / 2 \mathrm{Ry}-E_{\mathrm{g}}=$ $0.02401014046596021538(56) \mathrm{Ry}$ is practically the same as in [15], and close to the older estimate of $0.024010113 \mathrm{Ry}$ [12]. The effect of the corrections discussed above is to slightly decrease this energy to

$$
\begin{array}{r}
B_{1 e}^{\prime}=\left[0.02401014046596021538(56)-0.010773815602(8) \alpha^{2}\right. \\
\left.-0.3855457(9) \alpha^{3}\right] \mathrm{Ry}=0.02400941692485(6) \mathrm{Ry} .
\end{array}
$$


Table 6. Summary of relativistic and QED contributions to the binding energies of Ps and Ps ${ }^{-}$. Units are in Ry.

\begin{tabular}{llll}
\hline Term & $E_{\mathrm{Ps}}$ & $E_{\mathrm{Ps}^{-}}$ & $E_{\mathrm{Ps}^{-}}-E_{\mathrm{Ps}^{-}}$ \\
\hline$\left\langle H_{1}\right\rangle / \alpha^{2}$ & $-5 / 32$ & $-0.16125467393850(6)$ & $0.00500467393850(6)$ \\
$\left\langle H_{2}\right\rangle / \alpha^{2}$ & $1 / 4$ & $0.259466645837(8)$ & $-0.009466645837(8)$ \\
$\left\langle M_{2}\right\rangle / \alpha^{2}$ & $-1 / 4$ & $-0.2436881562960(1)$ & $-0.0063118437040(1)$ \\
$\left\langle H_{v p}\right\rangle / \alpha^{3}$ & $-1 /(15 \pi)$ & $-0.0220242129346(7)$ & $0.0008035538557(7)$ \\
$\delta_{1 p} E_{\mathrm{g}} / \alpha^{3}$ & $2.76687300(3)$ & $3.0064919(9)$ & $-0.2396189(9)$ \\
$\delta_{2 p} E_{\mathrm{g}} / \alpha^{3}$ & $-0.585335778(7)$ & $-0.510831605(7)$ & $-0.07450417(1)$ \\
$\delta_{2 \gamma} E_{\mathrm{g}} / \alpha^{3}$ & $-(1-\ln 2) / \pi$ & $-0.025448161055(1)$ & $-0.072226124976(1)$ \\
\hline
\end{tabular}

Worth noting, the observed ground-state splitting of positronium, sometimes called the hyperfine splitting [34] of $1.160963(9) \alpha^{2}$ Ry between the otherwise degenerate components $S_{13}=0$ and 1 , is due to an additional spin-spin contact interaction, which produces a level shift [34]

$\delta_{s}^{\mathrm{ep}} E_{n}^{0}=2 \pi \alpha^{2}\left\langle\delta\left(\vec{r}_{13}\right)\left\{\frac{4}{3} \vec{s}_{1} \cdot \vec{s}_{3}\left(1-\frac{\alpha}{2 \pi}\right)+\frac{1}{2} \vec{S}_{13}^{2}\left[1-\left(\frac{26}{9}+\ln 4\right) \frac{\alpha}{\pi}\right]\right\}\right\rangle_{n} f^{3}$ au.

This means a change in the energy of the singlet by $\delta_{s}^{\mathrm{ep}} E_{\mathrm{g}}^{0}=-2 \alpha^{2}(1-\alpha / 2 \pi) f^{3}$ au $=$ $-0.265947576(23) \times 10^{-4} \mathrm{Ry}$. In $\mathrm{Ps}^{-}$the corresponding variation of the ground-state level has two parts,

$$
\delta_{s}^{\mathrm{ee}} E_{\mathrm{g}}=-\frac{8 \pi}{3} \alpha^{2}\left(1+\frac{5}{2 \pi} \alpha\right)\left\langle\vec{s}_{1} \cdot \vec{s}_{2} \delta\left(\vec{r}_{12}\right)\right\rangle f^{3} \mathrm{au},
$$

arising from the electron-electron coupling [30], and

$$
\delta_{s}^{2 \mathrm{ep}} E_{\mathrm{g}}=\pi \alpha^{2}\left\langle\delta\left(\vec{r}_{13}\right)\left(\vec{S}_{13}^{2}+\vec{S}_{23}^{2}\right)\left[1-\left(\frac{26}{9}+\ln 4\right) \frac{\alpha}{\pi}\right]\right\rangle f^{3} \text { au }
$$

due to the two electron-positron terms. Together they add an energy shift $\delta_{s} E_{\mathrm{g}}=$ $\delta_{s}^{\text {ee }} E_{\mathrm{g}}+\delta_{s}^{2 \mathrm{ep}} E_{\mathrm{g}}=0.207196744(18) \times 10^{-4} \mathrm{Ry}$, and change $B_{1 \mathrm{e}}$ by $\delta_{s}^{\mathrm{ep}} E_{\mathrm{g}}^{0}-\delta_{s} E_{\mathrm{g}}=$ $-0.47314432(3) \times 10^{-4}$ Ry. Including the spin-spin contact terms, the one-electron binding energy becomes

$$
B_{1 \mathrm{e}}^{\prime \prime}=B_{1 \mathrm{e}}^{\prime}+\delta_{s}^{\mathrm{ep}} E_{\mathrm{g}}^{0}-\delta_{s} E_{\mathrm{g}}=0.023962102492(3) \mathrm{Ry} .
$$

The uncertainty due to terms of order $\alpha^{4}$ Ry and higher not included in the calculation is estimated to be $\pm 0.5 \alpha^{4} \mathrm{Ry}$.

\section{Summary and conclusions}

The calculation of the relativistic and QED corrections to the energy levels of a quantum threebody system represents a challenging problem, of fundamental importance in the atomic, and nuclear physics. Difficulties appear both at conceptual and computational levels, because there is no satisfactory relativistic many-body quantum theory, and the nonrelativistic problem is not integrable.

A quantum three-body system thoroughly investigated since the early days of quantum mechanics is the helium atom. In this case a major simplification occurs, because the reduced electron mass $\mu$ is smaller than the mass of the positive charge by a factor $1.3707 \times 10^{-4}$, and to a first approximation the motion of the nucleus in the centre-of-mass frame can be neglected. 
The relativistic invariance is partly restored by the Breit interaction, and highly accurate nonrelativistic wavefunctions can be obtained numerically, from variational calculations. Within this framework, a perturbative treatment of the relativistic and QED correction terms gives energy levels in remarkable agreement with experiment [6, 7].

The same procedure was applied in this work to the negative positronium ion. However, by contrast to helium, all three particles have equal mass, and a perturbative treatment of the positron motion becomes inappropriate.

The accuracy of the nonrelativistic energy and ground-state wavefunction was discussed in section 2. The extrapolated value $E_{\mathrm{g}}$ obtained here is $-0.26200507023298010769(28)$ au, the same as in $[15,13]$ and close within $10^{-8}$ to the estimates obtained by other methods [14]. The variance of the Hamiltonian for the largest (2528-dimensional) basis set is $2.78 \times 10^{-8}$ au, smaller than the level width $\Gamma_{\left(\mathrm{Ps}^{-}, 2 \gamma\right)}=\hbar \lambda_{\left(\mathrm{Ps}^{-}, 2 \gamma\right)}=5.06 \times 10^{-8}$ au due to the $(2 \gamma) \mathrm{e}^{-}$decay. To calculate the expectation values of complicated operators such as $H_{0}^{2}$, we have used a new procedure based on a decomposition into elementary functions suitable for automatic processing [36].

The calculation of the first relativistic and QED corrections has been presented in sections 3 and 4. Some of the most important matrix elements are given in tables 3 and 5 . The final results indicate that the first spin-independent relativistic terms contribute to the $\mathrm{Ps}^{-}$ground-state energy by $-0.072738092198(4) \alpha^{2}$ au and the lowest order QED corrections by $1.22409400(44) \alpha^{3}$ au. These terms decrease both the ground-state energy, to $E_{\mathrm{g}^{*}}=-0.2620084679599(4)$ au, and the one-electron binding energy from the nonrelativistic value $B_{1 \mathrm{e}}=-1 / 4 \mathrm{au}-E_{\mathrm{g}}$ to 0.01200470846243 (3) au. A much larger contribution appears however from the spin-dependent contact terms, which raise the groundstate energy to $E_{\mathrm{g}^{*}}=-0.261998108122$ (1) au, and further decrease the binding energy to $0.011981051246(2)$ au. Including the uncertainty due to terms of order $\alpha^{4}$ Ry and higher, the final result for the binding energy is $0.011981051(1) \mathrm{au}$, or $78831530 \pm 5 \mathrm{MHz}$. The calculated rate of spontaneous decay by two-photon emission is $2.092797(1) \mathrm{ns}^{-1}$, close to the previous theoretical results and to the measured value [2].

It would of course be very interesting to have a measurement of the binding energy of $\mathrm{Ps}^{-}$to provide a direct test of the quantum theory used to describe loosely bound three-body systems.

\section{Acknowledgments}

Financial support by the Natural Sciences and Engineering Research Council of Canada, and by SHARCnet is gratefully acknowledged.

\section{Appendix A. Expectation values of $p^{4}$}

The expectation values $\left\langle\nabla_{1}^{4}\right\rangle=\left\langle\nabla_{2}^{4}\right\rangle$ for the electrons in the $\mathrm{Ps}^{-}$ground state can be calculated numerically either by direct differentiation, or as $\left\langle\nabla_{1}^{4}\right\rangle_{E}=\left\langle\nabla_{1}^{4}+\nabla_{2}^{4}\right\rangle_{E} / 2=$ $\left\langle\left(\nabla_{1}^{2}+\nabla_{2}^{2}\right)^{2}\right\rangle_{E} / 2-\left\langle\nabla_{1}^{2} \nabla_{2}^{2}\right\rangle$, where the first term is expressed in the form

$$
\begin{aligned}
\frac{1}{2}\left\langle\left(\nabla_{1}^{2}+\nabla_{2}^{2}\right)^{2}\right\rangle_{E} & =2\left\langle\left(\tilde{E}_{\mathrm{g}}-\tilde{V}+\frac{1}{2} \nabla_{13} \cdot \nabla_{23}\right)^{2}\right\rangle \\
& =2\left[\tilde{E}_{\mathrm{g}}^{2}-2 \tilde{E}_{\mathrm{g}}\left\langle\tilde{V}-\frac{1}{2} \nabla_{13} \cdot \nabla_{23}\right\rangle+\left\langle\left(\tilde{V}-\frac{1}{2} \nabla_{13} \cdot \nabla_{23}\right)^{2}\right\rangle\right]
\end{aligned}
$$

( $\tilde{E}_{\mathrm{g}} \equiv E_{\mathrm{g}} / f \mathrm{au}$ ) by assuming that the variational ground state is practically eigenstate of $H_{0}$. Although in the limit $N_{\mathrm{b}} \rightarrow \infty$ should be the same, at finite $N_{\mathrm{b}}$ the numerical values obtained 
for $\left\langle\nabla_{1}^{4}\right\rangle$ and $\left\langle\nabla_{1}^{4}\right\rangle_{E}$ are slightly different. These estimates are given as a function of the basis size $N_{\mathrm{b}}$ in the first two columns of table 3 . The third column contains the corresponding expectation value for the positron,

$\left\langle\nabla_{3}^{4}\right\rangle_{E}=\left\langle\left(\nabla_{13}+\nabla_{23}\right)^{4}\right\rangle_{E}=2\left\langle\nabla_{13}^{4}\right\rangle_{E}+2\left\langle\nabla_{13}^{2} \nabla_{23}^{2}\right\rangle-8\left\langle\left(\tilde{E}_{\mathrm{g}}-\tilde{V}\right) \nabla_{13} \cdot \nabla_{23}\right\rangle$

\section{Appendix B. Error estimates}

The series of numerical values presented in tables 1 and 3 to 5 appear to be convergent, but for comparison with experiment, it is useful to also provide a single extrapolated value, representing the expected result of the calculation when $N_{\mathrm{b}} \rightarrow \infty$. The procedure adopted here to define this value depends on the manner of convergence. In the case of a sequence $\left\{f_{n}\right\}$ convergent as an alternating series, the extrapolated value $f_{\text {extp. }} \pm \sigma_{f}$, given in the last row, was defined as the arithmetic average of its last three consecutive terms, by $f_{\text {extp. }}=$ $\left(f_{n_{x}}+f_{n_{y}}+f_{n_{z}}\right) / 3, n_{x}<n_{y}<n_{z}$, and $\sigma_{f}^{2}=\left[\left(f_{n_{x}}-f_{\text {extp. }}\right)^{2}+\left(f_{n_{y}}-f_{\text {extp. }}\right)^{2}+\left(f_{n_{z}}-f_{\text {extp. }}\right)^{2}\right] / 3$. If $\left\{f_{n}\right\}$ approaches the limit by monotonous increase or decrease, then we assume that the series can be extended to infinity by the function $F(n)=f_{\text {extp. }}+A \mathrm{e}^{-\gamma n}$. The matching equations $F\left(n_{x}\right)=f_{n_{x}}, F\left(n_{y}\right)=f_{n_{y}}, F\left(n_{z}\right)=f_{n_{z}}$ between $F(n)$ and the last three calculated numerical values yield the parameter $f_{\text {extp. }}$ in the form [13]

$$
f_{\text {extp. }}=f_{n_{y}}+\frac{f_{n_{y}}-f_{n_{x}}}{R-1} .
$$

Here $R \equiv \mathrm{e}^{\gamma\left(n_{y}-n_{x}\right)}$ is the solution of the equation $R-1=R_{y}\left[1-R^{\left(n_{y}-n_{z}\right) /\left(n_{y}-n_{x}\right)}\right]$, where

$$
R_{y}=\frac{f_{n_{y}}-f_{n_{x}}}{f_{n_{z}}-f_{n_{y}}} .
$$

The error is measured by

$$
\sigma_{f}=\left|f_{n_{y}}-f_{\text {extp. }}\right|=\left|\frac{f_{n_{y}}-f_{n_{x}}}{R-1}\right| .
$$

If $n_{y}-n_{x}=n_{z}-n_{y}$, then $R=R_{y}$. When $n$ is simply $N_{\mathrm{b}}$, then $n_{y}-n_{x}=286$ is larger, but close to $n_{z}-n_{y}=252$, and $R=R_{y}$ still provides a reasonable estimate.

\section{Appendix C. QED corrections of order $\alpha^{3}$ in the limit $m_{3} \rightarrow \infty$}

When $m_{3} \rightarrow \infty(f=1)$ the vacuum polarization contribution and the part $\sim \alpha^{3}$ in $\delta_{s}^{\text {ee }} E_{n}$ of equation (63), $\left.\delta_{s}^{\text {ee }} E_{n}\right|_{\alpha^{3}}=-20\left\langle\vec{s}_{1} \cdot \vec{s}_{2} \delta\left(\vec{r}_{12}\right)\right\rangle_{n} \alpha^{3} / 3$ au, remain the same, but $X_{3}^{n}=0, Y_{i 3}^{n}=0$ and $\delta_{1 p} E_{n}$ becomes

$$
\begin{aligned}
\delta_{1 p} E_{n}^{\infty}=\alpha^{3}[ & \frac{4}{3}\left(\ln \frac{m c}{2 \hbar k_{R}}+\frac{5}{6}-\beta_{n}\right)\left\langle\delta\left(\vec{r}_{13}\right)+\delta\left(\vec{r}_{23}\right)\right\rangle_{n} \\
& \left.-\frac{8}{3}\left(\ln \frac{m c}{2 \hbar k_{R}}-\frac{1}{2}+\ln a_{\mu} k_{R}\right)\left\langle\delta\left(\vec{r}_{12}\right)\right\rangle_{n}-\frac{2}{3 \pi} Q_{12}^{n}\right] \text { au. }
\end{aligned}
$$

The two-photon exchange contribution reduces to

$$
\delta_{2 p} E_{n}^{\infty}=\alpha^{3}\left[-\frac{Q_{12}^{n}}{2 \pi}+2\left(\ln \alpha-\frac{4}{3} \ln 2+\frac{13}{6}\right)\left\langle\delta\left(\vec{r}_{12}\right)\right\rangle_{n}\right] \mathrm{au},
$$


and the total correction $\delta E_{n}^{\infty}=\delta_{1 p} E_{n}^{\infty}+\delta_{2 p} E_{n}^{\infty}+\left.\delta_{s}^{e e} E_{n}\right|_{\alpha^{3}}+\left\langle H_{v p}\right\rangle_{n}$ is

$$
\begin{aligned}
\delta E_{n}^{\infty}=\alpha^{3}\left[\frac{4}{3}\right. & \left(\frac{19}{30}-\ln \alpha^{2}-\beta_{n}\right)\left\langle\delta\left(\vec{r}_{13}\right)+\delta\left(\vec{r}_{23}\right)\right\rangle_{n} \\
& \left.+\left(\frac{14}{3} \ln \alpha+\frac{164}{15}\right)\left\langle\delta\left(\vec{r}_{12}\right)\right\rangle_{n}-\frac{7}{6 \pi} Q_{12}^{n}\right] \mathrm{au} .
\end{aligned}
$$

\section{References}

[1] Wheeler J A 1946 Ann. NY Acad. Sci. 48219

[2] Mills A P Jr 1981 Phys. Rev. Lett. 46717 Mills A P Jr 1983 Phys. Rev. Lett. 5067

[3] Ferrante G 1968 Phys. Rev. 17076

[4] Hylleraas E A 1928 Zeits.f. Phys. 48469 Hylleraas E A 1929 Zeits. f. Phys. 54347

[5] Drake G W F 1978 Phys. Rev. A 18820

[6] Drake G W F 1993 Long-Range Casimir Forces: Theory and Recent Experiments on Atomic Systems ed F S Levin and D A Micha (New York: Plenum) p 107

[7] Drake G W F 1996 Atomic, Molecular and Optical Physics Handbook ed G W F Drake (Woodbury: AIP Press) p 154

[8] Dirac P A M 1950 Can. J. Math. 2129

[9] Bethe H A and Salpeter E E 1977 Quantum Mechanics of One-and Two Electron Atoms (New York: Plenum Publishing Corporation) p 196

[10] Strange P 1998 Relativistic Quantum Mechanics (Cambridge: Cambridge University Press) p 322

[11] Ho Y K 1990 Phys. Lett. A 144237

[12] Bhatia A K and Drachman R J 1983 Phys. Rev. A 282523

[13] Drake G W F, Cassar M M and Nistor R A 2002 Phys. Rev. A 6554501

[14] Krivec R, Mandelzweig V B and Varga K 2000 Phys. Rev. A 6162503

[15] Frolov A M 1999 Phys. Rev. A 602834

[16] Ho Y K 1979 Phys. Rev. A 192347

Ho Y K 1997 Chin. J. Phys. 3597

[17] Ivanov I and Ho Y K 1999 Phys. Rev. A 601015 Ivanov I and Ho Y K 2000 Phys. Rev. A 61032501

[18] Usukura J and Suzuki Y 2002 Phys. Rev. A 6610502

[19] Igarashi A, Shimamura I and Toshima N 2000 New J. Phys. 217

[20] Bhatia A K and Drachman R J 1998 Nucl. Instrum. Methods Phys. Res. B 143195

[21] Ho Y K 1993 Phys. Rev. A 484780

[22] Messiah A 1961 Quantum Mechanics vol II, section 33 (Amsterdam: North-Holland)

[23] Stone A P 1961 Proc. Phys. Soc. (London) 77786 Stone A P 1963 Proc. Phys. Soc. (London) 81868

[24] Sapirstein J 1996 Atomic, Molecular and Optical Physics Handbook ed G W F Drake (Woodbury: AIP Press) p 327

[25] Bjorken J D and Drell S D 1964 Relativistic Quantum Mechanics (New York: McGraw Hill)

[26] Heisenberg W 1934 Z. Phys. 90209

[27] Uehling E A 1935 Phys. Rev. 4855

[28] Lamb W E Jr and Retherford R C 1947 Phys. Rev. 72241

[29] Fulton T and Martin P C 1954 Phys. Rev. 95811

[30] Araki H 1957 Prog. Theor. Phys. 17619

[31] Drake G W F and Goldman S P 1999 Can. J. Phys. 77835

[32] Pachucki K 1998 J. Phys. B: At. Mol. Opt. Phys. 315123

[33] Foot R and Gninenko S N 2000 Phys. Lett. B 480171

[34] Karplus R and Klein A 1952 Phys. Rev. 87848

[35] Therot E D Jr, Beers R H, Hughes V H and Ziock K O H 1970 Phys. Rev. A 2707

[36] Grigorescu M and Drake G W F 2003 LANL Preprint physics/0303095 